\title{
GENERALIZED LOCALIZATION FOR SPHERICAL PARTIAL SUMS OF MULTIPLE FOURIER SERIES
}

\author{
RAVSHAN ASHUROV
}

\begin{abstract}
In this paper the generalized localization principle for the spherical partial sums of the multiple Fourier series in the $L_{2}$ - class is proved, that is, if $f \in L_{2}\left(T^{N}\right)$ and $f=0$ on an open set $\Omega \subset T^{N}$, then it is shown that the spherical partial sums of this function converge to zero almost - everywhere on $\Omega$. It has been previously known that the generalized localization is not valid in $L_{p}\left(T^{N}\right)$ when $1 \leq p<2$. Thus the problem of generalized localization for the spherical partial sums is completely solved in $L_{p}\left(T^{N}\right), p \geq 1$ : if $p \geq 2$ then we have the generalized localization and if $p<2$, then the generalized localization fails.
\end{abstract}

AMS 2000 Mathematics Subject Classifications : Primary 42B05; Secondary 42B99. Key words: Multiple Fourier series, spherical partial sums, convergence almosteverywhere, generalized localization.

\section{INTRODUCTION}

Let $\left\{f_{n}\right\}, n \in Z^{N}$, be the Fourier coefficients of a function $f \in L_{2}\left(T^{N}\right), N \geq 2$. Consider the spherical partial sums of the multiple Fourier series:

$$
S_{\lambda} f(x)=\sum_{|n|^{2}<\lambda} f_{n} e^{i n x} .
$$

The aim of this paper is to investigate convergence almost-everywhere (a.e.) of these partial sums. One of the first questions which arise in the study of a.e. convergence of the sums (1.1) is the question of the validity of the Luzin conjecture: is it true that the spherical sums (1.1) of the Fourier series of an arbitrary function $f \in L_{2}\left(T^{N}\right)$ converge a.e. on $T^{N}$ ? In other words, does Carleson's theorem extend to $N$-fold Fourier series when the latter is summed spherically? The answer to this question is open so far. What is known is only that Hunt's theorem (convergence a.e. for $L_{p}$ functions) does not extend to $N$-fold $(N \geq 2)$ series summed by circles (see [1] and references therein). Historically progress with solving the Luzin conjecture has been made by considering easier problems. One of such easier problems is to investigate convergence a.e. of the spherical sums (1.1) on $T^{N} \backslash \operatorname{suppf}$.

Il'in 2] was the first to introduce the concept of generalized principle of localization for an arbitrary eigenfunction expansions. Following Il'in we say that the generalized localization principle for $S_{\lambda}$ holds in $L_{p}\left(T^{N}\right)$, if for any function $f \in L_{p}\left(T^{N}\right)$ the equality

$$
\lim _{\lambda \rightarrow \infty} S_{\lambda} f(x)=0
$$

holds a.e. on $T^{N} \backslash \operatorname{suppf}$.

Observe, unlike the classical Riemann localization principle, here it suffices the equality (1.2) to be hold only a.e. (not everywhere) on $T^{N} \backslash \operatorname{suppf}$.

For the spherical partial integrals of multiple Fourier integrals (we denote by $\sigma_{\lambda} f(x)$ ) the generalized localization principle in $L_{p}\left(R^{N}\right)$ has been investigated by many authors (see [3]9]). In particular, in the remarkable paper of A. Carbery and F. Soria [5] the validity of the generalized localization for $\sigma_{\lambda}$ has been proved in $L_{p}\left(R^{N}\right)$ when $2 \leq p<2 N /(N-1)$. Note, that the method introduced by these authors can be easily applied to non-spherical partial integrals too [10].

If we turn back to the multiple Fourier series (1.1) and consider the classes $L_{p}\left(T^{N}\right)$ when $1 \leq p<2$, then as A. Bastys [4] has proved, following Fefferman in making use of the Kakeya's 
problem, that the generalized localization for $S_{\lambda}$ is not valid, i.e. there exists a function $f \in$ $L_{p}\left(T^{N}\right)$, such that on some set of positive measure, contained in $T^{N} \backslash \operatorname{supp} f$, we have

$$
\varlimsup_{\lambda \rightarrow \infty}\left|S_{\lambda} f(x)\right|=+\infty .
$$

It may be worth mentioning that in 4 this result is also proved for the spherical partial integrals $\sigma_{\lambda} f(x)$.

The main result of this paper is the following statement.

Theorem 1.1. Let $f \in L_{2}\left(T^{N}\right)$ and $f=0$ on an open set $\Omega \subset T^{N}$. Then the equality (1.2) holds a.e. on $\Omega$.

Thus the problem of generalized localization for $S_{\lambda}$ is completely solved in classes $L_{p}\left(T^{N}\right)$, $p \geq 1$ : if $p \geq 2$ then we have the generalized localization and if $p<2$, then the generalized localization fails.

In the study of a.e. convergence it is convenient to introduce the maximal operator

$$
S_{\star} f(x)=\sup _{\lambda>0}\left|S_{\lambda} f(x)\right| .
$$

The prove of Theorem 1.1 is based on the following estimate of this operator.

Theorem 1.2. Let $f \in L_{2}\left(T^{N}\right)$ and $f=0$ on the ball $\{|x|<R\} \subset T^{N}$. Then for any $r<R$ there exists a constant $C=C(R, r)$, such that

$$
\int_{|x| \leq r}\left|S_{\star} f(x)\right|^{2} d x \leq C \int_{T^{N}}|f(x)|^{2} d x .
$$

The formulated theorems are easily transferred to the case of non-spherical partial sums of multiple Fourier series (see [10, [11]).

\section{Auxiliary assertions}

The proofs of Theorems 1.1 and 1.2 are based on several auxiliary assertions, which are given in this section. Here we have borrowed some original ideas from A. Carbery and F. Soria [5], where the authors have investigated the multiple Fourier integrals.

So we assume that $f=0$ on the fixed ball $\{|x|<R\} \subset T^{N}$ and fix a number $r<R$.

Let $\chi_{b}(t)$ be the characteristic function of the segment $[0, b]$. We denote by $\varphi_{1}(t)$ a smooth function with $\chi_{(R-r) / 3}(t) \leq \varphi_{1}(t) \leq \chi_{2(R-r) / 3}(t)$ and put $\varphi_{2}(t)=1-\varphi_{1}(t)$. Now we define a new function $\psi(x)$ as follows: $\psi(x)=\varphi_{2}(|x|)$, when $x \in T^{N}$ and otherwise it is a $2 \pi$ - periodical on each variable $x_{j}$ function.

Let us denote

$$
\theta(x, \lambda)=(2 \pi)^{-N} \sum_{|n|^{2}<\lambda} e^{i n x} .
$$

Then by definition of the Fourier coefficients we may write

$$
S_{\lambda} f(x)=\int_{T^{N}} \theta(x-y, \lambda) f(y) d y .
$$

If we define $\theta_{\lambda}(x)=\theta(x, \lambda) \psi(x)$, then we have

$$
S_{\lambda} f(x)=\int_{T^{N}} \theta_{\lambda}(x-y) f(y) d y, \text { for all } x, \text { with }|x| \leq r,
$$

since $f$ is supported in $\{|x| \geq R\}$. Therefore to prove the estimate (1.3) it suffices to obtain the inequality

$$
\int_{T^{N}} \sup _{q>0}\left|\theta_{q} * f\right|^{2} d x \leq C \int_{T^{N}}|f(x)|^{2} d x
$$

where sup is taken over all integers.

Now we need some estimates for the Fourier coefficients of the function $\theta_{k}(x)$, which we denote by $\left(\theta_{k}\right)_{n}$. 
Lemma 2.1. For an arbitrary integer $l$ there exists a constant $C_{l}$, depending on $l, r$ and $R$, such that for all $k \geq 0$ and $n \in Z^{N}$ one has

$$
\left|\left(\theta_{k}\right)_{n}\right| \leq \frac{C_{l}}{(1+|| n|-\sqrt{k}|)^{l}}
$$

Proof. Let $\left\{\psi_{m}\right\}$ be the Fourier coefficients of the function $\psi(x)$. Then

$$
\left(\theta_{k}\right)_{n}=(2 \pi)^{-2 N} \int_{T^{N}} \sum_{|m|<\sqrt{k}} e^{i m x} \psi(x) e^{-i n x} d x=(2 \pi)^{-N} \sum_{|n-m|<\sqrt{k}} \psi_{m} .
$$

If $|n|>\sqrt{k}$ then we have

$$
(2 \pi)^{-N}\left|\sum_{|n-m|<\sqrt{k}} \psi_{m}\right| \leq(2 \pi)^{-N} \sum_{|m|>|n|-\sqrt{k}}\left|\psi_{m}\right| .
$$

Similarly, if $|n| \leq \sqrt{k}$ then making use of the equality (observe, $\psi$ is an infinitely differentiable and $2 \pi$ - periodical function) $\sum \psi_{m}=\psi(0)=0$, we obtain

$$
(2 \pi)^{-N}\left|\sum_{|n-m|<\sqrt{k}} \psi_{m}\right|=(2 \pi)^{-N}\left|-\sum_{|n-m| \geq \sqrt{k}} \psi_{m}\right| \leq(2 \pi)^{-N} \sum_{|m| \geq \sqrt{k}-|n|}\left|\psi_{m}\right| .
$$

Now it is sufficient to note that for any integer $j \geq 0$ there exists a constant $c_{j}$, depending on $(R-r)$, such that

$$
\left|\psi_{m}\right| \leq \frac{c_{j}}{(1+|m|)^{j}}
$$

and to estimate the last sum by comparing it with the corresponding integral.

We will apply the estimate (2.2) further, so the corresponding constants will depend on $r$ and $R$. In addition, as we have done above, in order to estimate number series we compare them with the corresponding integrals.

Let $\left(\Theta_{j}\right)_{n}=\left(\theta_{j+1}\right)_{n}-\left(\theta_{j}\right)_{n}$, that is,

$$
\left(\Theta_{j}\right)_{n}=(2 \pi)^{-N} \sum_{|m|^{2}=j} \psi_{m-n}=(2 \pi)^{-N} \sum_{|n-m|^{2}=j} \psi_{m}
$$

(if the Diophantine equation $|m-n|^{2}=j$ does not have a solution, then $\left.\left(\Theta_{j}\right)_{n}=0\right)$. These numbers have a better estimate than $\left(\theta_{j}\right)_{n}$ in the following sense. Suppose $k \leq \sqrt{j}<k+1$, i.e. $k^{2} \leq j<k^{2}+2 k+1$, or $j=k^{2}+p, 0 \leq p<2 k+1$, then according to Lemma 2.1] $\left(\theta_{j}\right)_{n}$ has the same estimate. But, as we will see below, the numbers $\left(\Theta_{j}\right)_{n}$ vanish in the same interval in some sense. In particular, the following statement is true.

Lemma 2.2. For any $l$, there exists a constant $C_{l}$ such that

$$
\sum_{k \leq \sqrt{\jmath}<k+1}\left|\left(\Theta_{j}\right)_{n}\right|^{2} \leq \frac{C_{l}}{(1+|| n|-k|)^{l}} .
$$

Proof. Let $|n| \leq k$; otherwise estimates are similar. By virtue of estimate (2.2) we have

$$
\begin{aligned}
& \sum_{k \leq \sqrt{j}<k+1}\left|\left(\Theta_{j}\right)_{n}\right| \leq(2 \pi)^{-N} \sum_{k \leq|n-m|<k+1}\left|\psi_{m}\right| \leq \\
& \leq(2 \pi)^{-N} \sum_{|m|>|| n|-k|} \frac{c_{j}}{(1+|m|)^{j}} \leq \frac{C_{l}}{(1+|| n|-k|)^{l}} .
\end{aligned}
$$

Since $\left|\left(\Theta_{j}\right)_{n}\right|^{2} \leq C\left|\left(\Theta_{j}\right)_{n}\right|$, Lemma is proved.

Corollary 2.3. Uniformly on $n$ one has

$$
\sum_{j=0}^{\infty}\left|\left(\Theta_{j}\right)_{n}\right|^{2}=\sum_{k=0}^{\infty} \sum_{k \leq \sqrt{j}<k+1}\left|\left(\Theta_{j}\right)_{n}\right|^{2} \leq C .
$$


If we properly group the numbers $\left(\Theta_{k^{2}+p}\right)_{n}$ by parameter $p$, then a stronger result than Lemma 2.2 can be obtained. Our nearest aim is to implement this grouping.

Denote by $y_{0}$ (the nearest one to the origin) the intersection point of the ball $\left\{x \in R^{N}\right.$ : $|x-n| \leq k+1\}$ with the straight line $O n$ that passes through the origin and point $n$. Let $T_{y_{0}}$ be the tangential hyperplane to the ball $\left\{x \in R^{N}:|x-n| \leq k+1\right\}$ at the point $y_{0}$. Let $B_{0}:=$ $\left\{y \in T_{y_{0}}:\left|y-y_{0}\right|<1\right\}$ and $B_{j}:=\left\{y \in T_{y_{0}}: \sqrt{j} \leq\left|y-y_{o}\right|<\sqrt{j+1}\right\}$, where $j=1,2, \cdots, 2 k-1$. Let $C_{j}^{k}, j=0,1, \cdots, 2 k-1$, be the $N$ - dimensional cylinders with the base $B_{j}$ and with the axis parallel to $O n$ and the length $|n|$. Consider the ring $K=\left\{x \in R^{N}: k \leq|x-n|<(k+1)\right\}$ and divide it in to the following sets: $P_{j}^{k}=K \cap C_{j}^{k}, j=0,1, \cdots, 2 k-1$.

Let us define the sets $Q_{q}^{k}, q=0,1, \cdots, 2 k-1$, as follows. Let $Q_{q}^{k}$ be the set of those integers $p, 0 \leq p \leq 2 k$, for which the Diophantine equation $|m-n|^{2}=k^{2}+p$ has a solution in $P_{q}^{k}$. If $P_{q}^{k}$ does not contain any of solutions of equation $|m-n|^{2}=k^{2}+p$, for any $p$, then we assign to the set $Q_{q}^{k}$ one of those parameters $p$ that are not included in the previous sets $Q_{j}^{k}, j=0,1, \cdots, q-1$. If there are no such $p^{\prime} s$ left, then we define $Q_{j}^{k}, j=q, q+1, \cdots, 2 k-1$ as empty set.

In the proof of Lemma 2.7 we need to know how many at most parameters $p$ does the set $Q_{q}^{k}$ contain. Observe, if we fix $y \in O n$, then the Diophantine equation $m \in Z^{N},|m-y|^{2}=$ $t, q \leq t<q+1$ may have a solution only for one $t$ (note, in fact, here it suffices to consider the "projection" of this equation onto the hyperplane passing through the point $y$ and parallel to $T_{y_{0}}$ ). The length of the projection of $P_{q}^{k}$ on the axis of $O x_{1}$ is less than $2 \sqrt{q+1}$; (without loss of generality, we can assume that the angle between $O n$ and $O x_{1}$ is less than or equal to $\frac{\pi}{4}$ ). Consequently, if, for a fixed $p$, there is a solution of the Diophantine equation $|m-n|^{2}=k^{2}+p$, provided $m \in P_{q}^{k}$, then the first coordinates $m_{1}$ of the numbers $m$, take less than $[2 \sqrt{q+1}]([a]$ is the integer part of the number $a$ ) different values. When $p$ varies from 0 to $2 k$, then each of these numbers $m_{1}$ can take at most two adjacent integer numbers. Hence each set $Q_{q}^{k}$ has less than $4 \sqrt{q+1}$ parameters $p$ with the above property .

With this choice of $Q_{q}^{k}$ we have the following statement.

Lemma 2.4. Let $q=0,1, \cdots, 2 k-1$ and $S_{p}=\left\{m \in Z^{N}:|m-n|^{2}=k^{2}+p\right\} \quad(p=0,1, \cdots, 2 k)$. If $|n| \geq k+1$, then

$$
\min _{m \in S_{p}, p \in Q_{q}^{k}}|m| \geq \sqrt{(|n|-k-1)^{2}+q}
$$

If $k<|n|<k+1$, then

$$
\min _{m \in S_{p}, p \in Q_{q}^{k}}|m| \geq \sqrt{q}
$$

If $|n| \leq k$, then

$$
\min _{m \in S_{p}, p \in Q_{q}^{k}}|m| \geq \frac{1}{2} \sqrt{(|n|-k)^{2}+q} .
$$

Proof. Note that it is sufficient to estimate the minimum distance from the origin to the set $P_{q}^{k}$. If $|n| \geq k+1$, then it is not hard to verify that the distance from the origin to the set $B_{q}$ is equal to $\sqrt{(|n|-k-1)^{2}+q}$. Obviously, this value is less or equal to the distance between the origin and $P_{q}^{k}$. In case of $k<|n|<k+1$ arguments are similar.

If $|n| \leq k$, then minimum distance from the origin to the set $P_{q}^{k}$ is less than or equal to $\sqrt{\left(|n|-\sqrt{k^{2}-q}\right)^{2}+q}$. But we can estimate this number from below by $\frac{1}{2} \sqrt{(|n|-k)^{2}+q}$.

As we mentioned above for $\left(\Theta_{j}\right)_{n}$ one has a more stronger result than Lemma 2.2

Lemma 2.5. For any $l$, there exists a constant $C_{l}$ such that

$$
\sum_{q=0}^{2 k-1}(q+1)^{2} \sum_{p \in Q_{q}^{k}}\left|\left(\Theta_{k^{2}+p}\right)_{n}\right|^{2} \leq \frac{C_{l}}{(1+\sqrt{|| n|-k|})^{l}} .
$$

Proof. From the definition of $\left(\Theta_{j}\right)_{n}$ one has

$$
\sum_{q=0}^{2 k-1}(q+1) \sum_{p \in Q_{q}^{k}}\left|\left(\Theta_{k^{2}+p}\right)_{n}\right| \leq(2 \pi)^{-N} \sum_{q=0}^{2 k-1}(q+1)^{2} \sum_{p \in Q_{q}^{k}} \sum_{|m-n|^{2}=k^{2}+p}\left|\psi_{m}\right| \leq
$$


(and by virtue of estimates (2.2) and (2.4) (we assume that $|n| \geq k+1$; otherwise arguments are similar) we finally have)

$$
\leq \sum_{q=0}^{2 k-1}(q+1)^{2} \sum_{|m| \geq \sqrt{(|n|-k-1)^{2}+q}} \frac{c_{j}}{(1+|m|)^{j}} \leq \frac{C_{l}}{(1+\sqrt{|| n|-k|})^{l}} .
$$

Now (2.5) follows from the estimate $\left|\left(\Theta_{j}\right)_{n}\right|^{2} \leq C\left|\left(\Theta_{j}\right)_{n}\right|$.

Next statement is an easy consequence of this Lemma.

Corollary 2.6. Uniformly on $n$, one has

$$
\sum_{k=0}^{\infty} \sum_{q=0}^{2 k-1}(q+1)^{2} \sum_{p \in Q_{q}^{k}}\left|\left(\Theta_{k^{2}+p}\right)_{n}\right|^{2} \leq C .
$$

Now we turn back to the Fourier coefficients $\left(\theta_{j}\right)_{n}$. From Lemma 2.1 we have the following estimate.

Lemma 2.7. Uniformly on $n$, one has

$$
\sum_{k=0}^{\infty} \sum_{q=0}^{2 k-1}(q+1)^{-2} \sum_{p \in Q_{q}^{k}}\left|\left(\theta_{k^{2}+p}\right)_{n}\right|^{2} \leq C
$$

Proof. As we mentioned above, each $Q_{q}^{k}$ has less than $4 \sqrt{q+1}$ parameter $p$. Therefore, by virtue of Lemma 2.1 one has

$$
\sum_{k=0}^{\infty} \sum_{q=0}^{2 k-1}(q+1)^{-2} \sum_{p \in Q_{q}^{k}}\left|\left(\theta_{k^{2}+p}\right)_{n}\right|^{2} \leq \sum_{k=0}^{\infty} \frac{C_{l}}{(1+|| n|-k|)^{l}} \sum_{q=0}^{2 k-1} \frac{4 \sqrt{q+1}}{(q+1)^{2}} \leq C .
$$

\section{Proofs of Theorems}

First, we prove the estimate (2.1). Let $\Theta_{j}(x)=\theta_{j+1}(x)-\theta_{j}(x)$. Then $\theta_{j+1} * f+\theta_{j} * f=$ $2 \theta_{j} * f+\Theta_{j} * f$. Note the Fourier coefficients of the function $\Theta_{j}(x)$ are the numbers $\left(\Theta_{j}\right)_{n}$, introduced above.

If for a sequence of numbers $\left\{F_{q}\right\}$ we have $F_{0}=0$, then

$$
F_{q}^{2}=\sum_{j=0}^{q-1}\left[F_{j+1}-F_{j}\right]\left[F_{j+1}+F_{j}\right], q \geq 1
$$

Hence

or

$$
\left[\theta_{q} * f\right]^{2}=\sum_{j=0}^{q-1}\left[\Theta_{j} * f\right]^{2}+2 \sum_{j=0}^{q-1}\left[\Theta_{j} * f\right]\left[\theta_{j} * f\right]
$$

$$
\sup _{q>0}\left|\theta_{q} * f\right|^{2} \leq \sum_{j=0}^{\infty}\left|\Theta_{j} * f\right|^{2}+2 \sum_{k=0}^{\infty} \sum_{q=0}^{2 k-1} \sum_{p \in Q_{q}^{k}}\left|\Theta_{k^{2}+p} * f\right|(q+1)\left|\theta_{k^{2}+p} * f\right|(q+1)^{-1}
$$

Integrating over $T^{N}$ and making use of the inequality $2 a b \leq a^{2}+b^{2}$ one has

$$
\begin{gathered}
\int_{T^{N}} \sup _{q>0}\left|\theta_{q} * f\right|^{2} \leq \sum_{n}\left|f_{n}\right|^{2} \sum_{j=0}^{\infty}\left|\left(\Theta_{j}\right)_{n}\right|^{2}+ \\
+\sum_{n}\left|f_{n}\right|^{2} \sum_{k=0}^{\infty} \sum_{q=0}^{2 k-1}(q+1)^{2} \sum_{p \in Q_{q}^{k}}\left|\left(\Theta_{k^{2}+p}\right)_{n}\right|^{2}+ \\
+\sum_{n}\left|f_{n}\right|^{2} \sum_{k=0}^{\infty} \sum_{q=0}^{2 k-1}(q+1)^{-2} \sum_{p \in Q_{q}^{k}}\left|\left(\theta_{k^{2}+p}\right)_{n}\right|^{2} \leq
\end{gathered}
$$


(making use of Corollaries 2.3 and 2.6 and Lemma 2.7 and since $f$ is $L_{2}$ - function)

$$
\leq C \sum_{n}\left|f_{n}\right|^{2}=C \int_{T^{N}}|f(x)|^{2} d x .
$$

Thus, the estimate (2.1) and, consequently, Theorem [1.2 is proved.

Now we prove Theorem 1.1 So let $f \in L_{2}\left(T^{N}\right)$ and $f=0$ on an open set $\Omega \subset T^{N}$. We extend $f(x)$ to outside of $T^{N} 2 \pi$ - periodically on each variable $x_{j}$. In these conditions we must prove that the equality (1.2) holds a.e. on $\Omega$. If $x \in \Omega$ an arbitrary point, then to do this it suffices to show validity of (1.2) a.e. on a ball with center at $x$ and sufficiently small radius $R$, so that this ball belongs to $\Omega$. Therefore without loss of generality we may suppose, that $f$ is supported outside of this ball or by translation invariance, $f$ is supported in $\{|x| \geq R\}$, and prove convergence to zero of $S_{\lambda} f(x)$ a.e. on the ball $\{|x|<r\}$ for any $r<R$. But this statement can be proved by a standard technique based on Theorem 1.2 (see [12]). Thus Theorem 1.1 is also proved.

\section{ACKNOWLEDGEMENT}

The author conveys thanks to Sh. A. Alimov for discussions of this result and gratefully acknowledges Marcelo M. Disconzi (Vanderbilt University, USA) for support and hospitality.

The author was supported by Foundation for Support of Basic Research of the Republic of Uzbekistan (project number is OT-F4-88).

\section{REFERENCES}

1. Alimov, Sh.A., Ashurov, R.R., Pulatov, A.K.: Multiple Fourier Series and Fourier Integrals. Commutative Harmonic Analysis, vol. IV, pp. 197. Springer, Berlin (1992)

2. Il'in, V.A.: On a generalized interpretation of the principle of localization for Fourier series with respect to fundamental systems of functions, Sib. Mat. Zh., 9, 1093-1106 (1968)

3. Bastys, A.J.: The generalized localization principle for an N-fold Fourier integral, Dokl. Akd. Nauk SSSR, 278, 777-778 (1984)

4. Bastys, A.J.: Generalized localization of Fourier series with respect to the eigenfunctions of the Laplace operator in the classes Lp, Litovskii Matematicheskii Sbornik, 31, 387-405 (1991)

5. Carbery, A., Soria F.: Almost everywhere convergence of Fourier integrals for functions in Sobolev spaces, and an $L_{2}$-localization principle, Revista Mat. Iberoamericana 4, 319- 337 (1988)

6. Carbery, A., Rubio de Francia, J. L., Vega, L.: Almost everywhere summability of Fourier integrals, J. London Math. Soc. 38, 513-524 (1988)

7. Carbery, A., Romera, E., Soria, F.: Radial weights and mixed norm inequalities for the disc multiplier, J. Funct. Anal. 109, 52-75 (1992)

8. Carbery, A. Soria, F.: Pointwise Fourier inversion and localization in $R^{n}$, J. Fourier Anal. Appl., 3, Special Issue, 847-858 (1997)

9. Sjölin, P.:Regularity and integrability of spherical means, Monatsh. Math. 96, 277-291 (1983)

10. Ashurov,R. R., Ahmedov,A., Ahmad Rodzi b. Mahmud,:The generalized localization for multiple Fourier integrals, J. Math. Anal. Appl., 371 832-841(2010)

11. Ashurov, R.R., Butaev, A.: On the Pinsky phenomenon, J. Fourier Anal. Appl. 16, 804-812 (2010)

12. Stein, E.M.,Weiss, G.: Itroduction to Fourier analysis on Euclidean spaces, Princeton University Press, Princeton, New Jersey, (1971)

National University of Uzbekistan named after Mirzo Ulugbek and Institute of Mathematics, Uzbekistan Academy of Science

Current address: Institute of Mathematics, Uzbekistan Academy of Science, Tashkent, 81 Mirzo Ulugbek str. 100170

E-mail address: ashurovr@gmail.com 